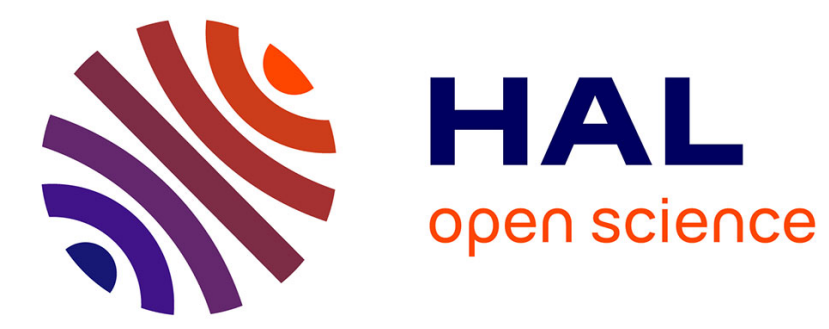

\title{
TRANSPORT PROPERTIES OF HEAVY ELECTRON METALS
}

D. Jaccard, J. Sierro, J. Brison, J. Flouquet

\section{To cite this version:}

D. Jaccard, J. Sierro, J. Brison, J. Flouquet. TRANSPORT PROPERTIES OF HEAVY ELECTRON METALS. Journal de Physique Colloques, 1988, 49 (C8), pp.C8-741-C8-746. 10.1051/jphyscol:19888337 . jpa-00228513

\section{HAL Id: jpa-00228513 https://hal.science/jpa-00228513}

Submitted on 1 Jan 1988

HAL is a multi-disciplinary open access archive for the deposit and dissemination of scientific research documents, whether they are published or not. The documents may come from teaching and research institutions in France or abroad, or from public or private research centers.
L'archive ouverte pluridisciplinaire HAL, est destinée au dépôt et à la diffusion de documents scientifiques de niveau recherche, publiés ou non, émanant des établissements d'enseignement et de recherche français ou étrangers, des laboratoires publics ou privés. 


\title{
TRANSPORT PROPERTIES OF HEAVY ELECTRON METALS
}

\author{
D. Jaccard $\left({ }^{1}\right)$, J. Sierro $\left({ }^{1}\right)$, J. P. Brison $\left({ }^{2}\right)$, and J. Flouquet \\ $\left({ }^{1}\right)$ Département de Physique de la matière Condensée, Genève, Suisse \\ $\left(^{2}\right)$ Centre de Recherche sur les Très Basses Températures, CNRS, B.P. 166X, 3804̨2 Grenoble Cedex, France
}

\begin{abstract}
Resistivity $(\rho)$ and thermoelectric power $(Q)$ experiments are described for two different ground states at zero pressure $(P)$ : a normal phase $\left(\mathrm{CeAl}_{3}\right)$ and a superconducting phase $\left(\mathrm{UBe}_{13}\right)$. In $\mathrm{CeAl}_{3}$, antiferromagnetism occurs clearly at $P=0$ and may disappear rapidly under pressure. Magnetic field and pressure experiments in UBe 13 show that antiferromagnetism and superconductivity may be antagonist.
\end{abstract}

\section{Introduction.}

Recent measurements in heavy fermion metals underline the importance of the magnetism. For example, the previous classification of the ground state as a normal Pauli paramagnetic phase in $\mathrm{CeAl}_{3}$ or a superconducting and non magnetic phase in $\mathrm{CeCu}_{2} \mathrm{Si}_{2}$, is now revised; both of these compounds seem to undergo an antiferromagnetic order with a tiny ordered moment suggesting itinerant magnetism [1]. Indeed most heavy fermion superconductors ( $\mathrm{URu}_{2} \mathrm{Si}_{2}, \mathrm{CeCu}_{2} \mathrm{Si}_{2}, \mathrm{UPt}_{3}$, $\mathrm{U}_{1-x} \mathrm{Th}_{x} \mathrm{Be}_{13}$ with $\left.x<0.015\right)[2-4]$ are now considered as antiferromagnetic. Inelastic neutron measurements on (yet assumed heavy fermion paramagnets), $\mathrm{CeCu}_{6}$ and $\mathrm{CeRu}_{2} \mathrm{Si}_{2}$ show the coexistence of antiferromagnetic correlations and strong local spin fluctuations. Their relative weights may be monitored by applied magnetic field $(H)$ or pressure $(P)[5,6]$.

Low ordered magnetic moments have been mainly detected by muon spin relaxation ( $\mu \mathrm{SR})$ [4], nuclear magnetic resonance (NMR) [2] or neutron scattering experiments when large single crystals can be realized as for $\mathrm{UPt}_{3}$ [3]. Here, focus will be given on transport measurements realized on a non superconducting $\left(\mathrm{CeAl}_{3}\right)$ and a superconducting ( $\left.\mathrm{UBe}_{13}\right)$ compound. The reported magnetoresistivity data are obtained on very small single crystals of $\mathrm{CeAl}_{3}$. Details about samples and experimental techniques can be found in reference [7-9]. Measurements on $\mathrm{UBe}_{13}$ were performed down to $50 \mathrm{mK}$ in magnetic field up to $10 \mathrm{~T}$ at very high pressure $(P \leq 80 \mathrm{kbar})$ generated by a pair of opposed Bridgman anvils made of non magnetic tungsten carbide. Due to the large value of the upper critical field $H_{\mathrm{c} 2}(T=0) \sim 13.9 \mathrm{~T}$ of $\mathrm{UBe}_{13}$ the high magnetic fields of the Service National des Champs Intenses and the Max-Planck Institut of Grenoble were used to study the resistivity $(\rho)$ of the normal phase [10].
$\mathrm{CeAl}_{3}$ : an antiferromagnet with tiny ordered magnetic moments?

$\mu \mathrm{SR}$ experiments down to $35 \mathrm{mK}$ lead to the observation of a spontaneous muon spin precession frequency in zero external field below $0.7 \mathrm{~K}$ and of a non oscillatory component which collapses only above $1.5 \mathrm{~K}$. Clearly, a quasistatic internal magnetic field of finite average value occurs below $0.7 \mathrm{~K}$ and below $1.5 \mathrm{~K}$ magnetic correlations seem to set up [11].

In the same time [7], resistivity measurements on single crystals of $\mathrm{CeAl}_{3}$ show unusual behaviours not yet detected on polycrystals. From $300 \mathrm{~K}$ to $10 \mathrm{~K}$, $\rho_{\|}$and $\rho_{\perp}$ i.e. parallel and perpendicular to the $c-$ axis of the hexagonal structure, exhibit a constant large anisotropy with a ratio $\rho_{\perp} / \rho_{\|} \sim 3$. The average $\left(\langle\rho\rangle=1 / 3\left(\rho_{\|}+2 \rho_{\perp}\right)\right)$ is in excellent agreement with experimental data obtained for polycrystalline samples made from the same starting materials. The weak temperature dependence of $\rho_{\perp} / \rho_{\|}$well above the CF splitting $(\Delta \sim 90 \mathrm{~K})$ indicates that the anisotropy of the mixing interaction must be taken into account in theoretical models.

At low temperature, the resistivity of the single crystals differs markedly from that of polycrystals. Evidences of magnetic order have been found i) at $1.6 \mathrm{~K}$ where $\rho_{\|}$shows a break while no anomaly occurs in $\rho_{\perp}$ and ii) for $0.4<T<1 \mathrm{~K}$, where both $\rho_{\|}$and $\rho_{\perp}$ have $T^{3}$ dependences. $T^{n}$ power laws with $n>2$ are reminiscent of electron-magnon scattering; for example, they are observed in the antiferromagnetic ordered Kondo compound $\mathrm{CeAl}_{2}$ [12]. In this temperature range, the polycrystalline samples have a quasilinear $T$ resistivity [13].

The obtention of single crystals gives the possibility of significant magnetoresistivity experiments as realized on $\mathrm{CeRu}_{2} \mathrm{Si}_{2}$ [14]. The amplitude of the magnetoresistivity is coupled to that of the magnetization 


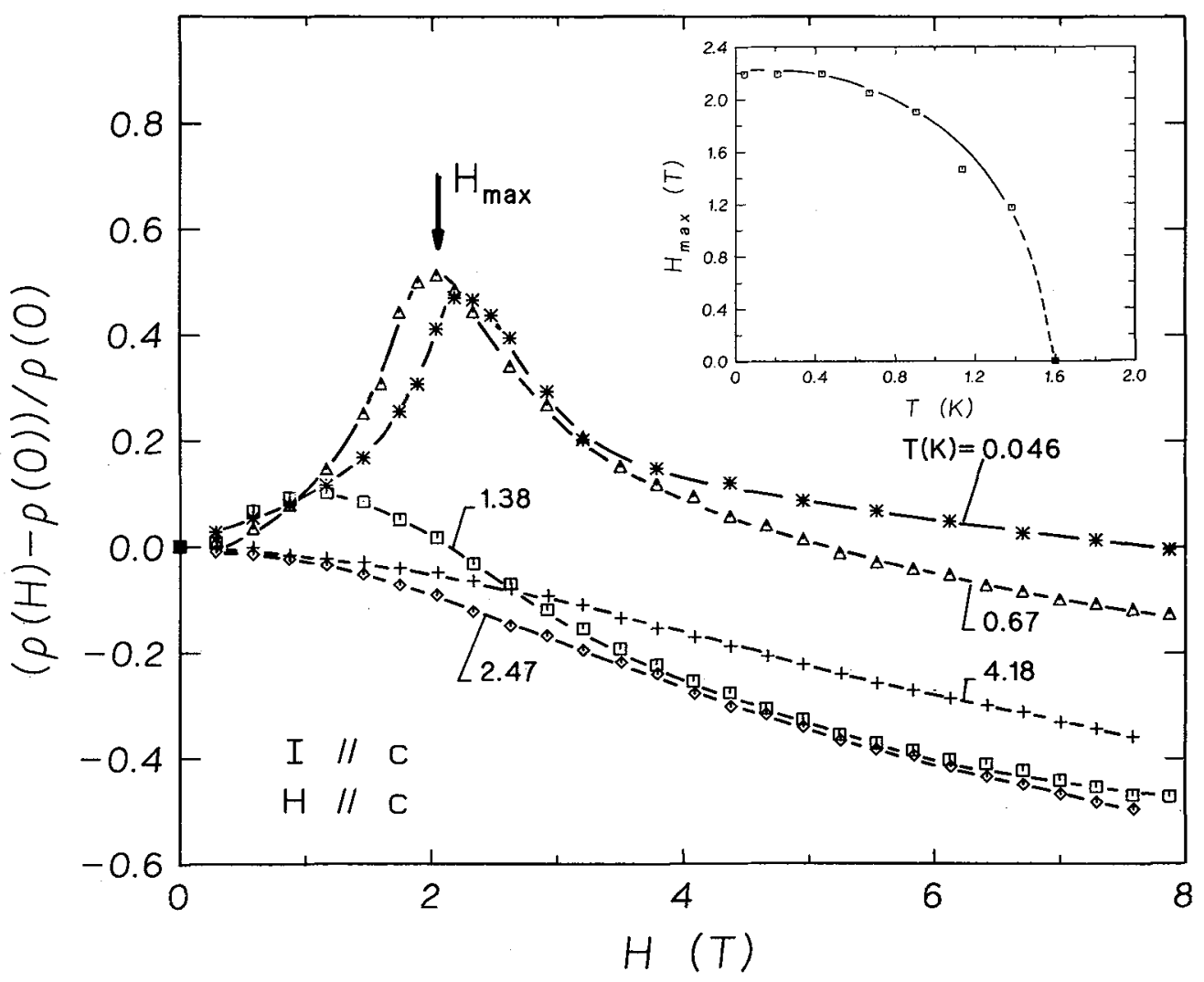

Fig. 1. - Longitudinal magnetoresistance along the $c$ axis of $\mathrm{CeAl}_{3}$ at different low temperatures. Insert is the magnetic phase diagram deduced from the ternperature dependence of $H_{\max }$, the maximum of the isothermal magnetoresistivity.

which is induced by the magnetic field. In agreement with the observation that the $c$-axis is the easy magnetization axis, the magnetoresistivity is large only when the magnetic field is parallel to the $c$-axis. As shown in figure 1 , below $1.6 \mathrm{~K}$, an initial positive magnetoresistivity appears up to a magnetic field $H_{\max }$. The temperature dependence of $H_{\max }$ shown in the insert is strongly analogous to $\mathrm{H}, \mathrm{T}$ phase diagrams of magnetic systems.

Below $0.35 \mathrm{~K}, \rho_{\|}$and $\rho_{\perp}$ follow the standard law $\rho(H)=\rho_{0}(H)+A(H) T^{2}$, with $\rho_{0 \sharp}=14.5 \mu \Omega \mathrm{cm}$, $\rho_{0 \perp}=9.9 \mu \Omega \mathrm{cm}$, and $A_{\|}=4.6 \mu \Omega \mathrm{cm} / \mathrm{K}^{2}, A_{\perp}=$ $12.8 \mu \Omega \mathrm{cmK}^{-2}$. Figure 2 shows that the magnetic field dependence of $A_{\|}(H)$ has a narrow peak at $H \sim 2 \mathrm{~T}$ i.e. nearly at the crossover field $H_{\max }$. Such a coincidence is found at the metamagnetic like transition of $\mathrm{CeRu}_{2} \mathrm{Si}_{2}$ [14]. It is worthwhile to mention that non linearity of the magnetization with $H$ was already reported in the pionnering work of reference [15]. In polycrystals, field maxima of $\Delta \rho / \rho, \rho_{0}(H)$ and $A(H)$ have been reported with the same field $H_{\max }$ but with lower magnitude corresponding roughly to the random projection of an hexagonal magnetoresistance tensor. The occurrence of maxima in the magnetoresistivity

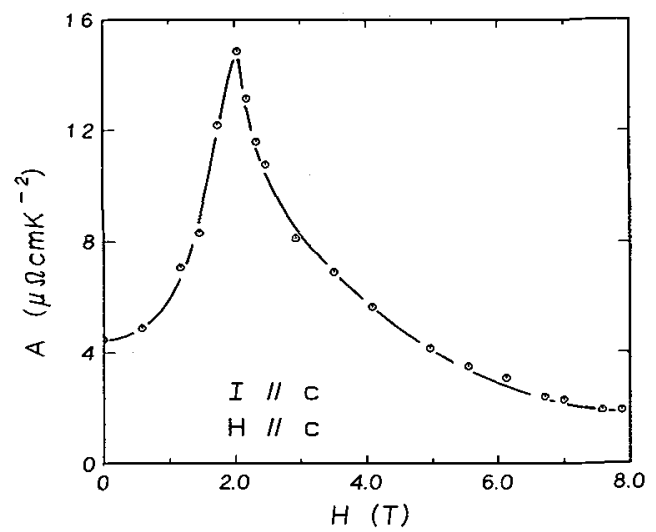

Fig. 2. - Magnetic field dependence of the $A_{\|}(H)$ coefficient in the lax $\rho_{\|}=\rho_{0 \|}+A_{\|} T^{2}$ of $\mathrm{CeAl}_{3}$.

are predicted for anisotropic weakly antiferromagnets [16]. Evidence of magnetic order is also given by the NMR observation that the NMR linewidth increases markedly below $1.2 \mathrm{~K}$ and the nuclear spin lattice relaxation rate $1 / T_{1}$ exhibits a sharp maximum also at 
$1.2 \mathrm{~K}[2,17]$. The initial increase of $A(H=0)$ with $P$ in contrast to the rapid decrease with $P$ above 3 kbar is interpreted as a transition from an antiferromagnetic lattice to a Pauli paramagnet [18].

The values of $\rho_{0}$ obtained in these crystals are far higher than those reached in polycrystals ( $\rho_{0} \leq$ $2 \mu \Omega \mathrm{cm}$ [15-18]). For $\rho_{0} \leq 2 \mu \Omega \mathrm{cm}$, quasiconstant determinations of $A=33 \bar{\mu} \Omega \mathrm{cmK}^{-2}$ are reported in polycrystals [15-18]. The large dependence of $A$ with $\rho_{0}$ (comparison between polycrystals and single crystals) suggests that $A$ cannot be entirely attributed to intrinsic interactions between quasiparticles built in a perfect lattice. An hypothesis to explain this unusual interference between $\rho_{0}$ and $A$ is that impurities behave like "Kondo holes" highly sensitive to any change of the Kondo lattice [8]. As it is yet difficult to control the perfection of the lattice, one way to explore the interplay between lattice and impurities is to probe their relative sensitivity to the pressure $(P)$ variable. Extensive $P$ resistivity experiments [18] show that large variations of $A(P)$ are also attended by a strong $P$ variation of $p_{0}$. Magnetic field or pressure dependence of the residual resistivity reflects the microscopic change in the interactions between the quasiparticles of the $\mathrm{CeAl}_{3}$ lattice.

If this notion of "Kondo holes" is widely used in heavy Fermion systems particularly to discuss their superconducting properties at low temperature [19-21], few theoretical works $[22,23]$ concern the normal state. The strong correlations between $\rho_{0}$ and $A$ lead to ask the crucial question of the influence of disorder on the nature of the ground state. The observation of tiny ordered moments supports a picture where f electrons are itinerant so that their physical properties (for example connected with nesting) may depend strongly on doping. As the occurrence of tiny ordered moments seems just now established, there is yet no systematic work which may give a clear answer. For $\mathrm{CeAl}_{3}$, as similar field dependences of $A(H)$ and $\rho_{0}(H)$ are observed for samples with substantial differences in $\rho_{0}$, no drastic change should occur here on the nature of the ground state.

\section{$\mathrm{UBe}_{13}$ : interplay between superconductivity and magnetism}

$\mathrm{UBe}_{13}$ is a fascinating heavy Fermion compound as superconductivity appears for a temperature $T_{c}$ where no Fermi liquid behaviour has still been achieved i.e. the characteristic temperature $T^{*}$ of the normal ground state is comparable to $T_{\mathrm{c}}$. The remarkable experimental facts at $P=0$ are i) in the normal phase an unusual large resistivity just above $T_{c}(\sim 150 \mu \Omega \mathrm{cm})$ [24] and a thermoelectric power $Q$ which has even not reached its negative maximum $(\sim-20 \mu \mathrm{V} / \mathrm{K})$ at $T^{Q_{\min }}$ when superconductivity sets up at $T_{c} \sim 950 \mathrm{mK}$
[25] and ii) in the superconducting state, the possible occurrence of large critical fluctuations $[10,21]$ and evidences for an axial pairing with an energy gap having point nodes on the Fermi surface [27].

The high pressure thermoelectric power $Q(P)$ data of figure 3 show the emergence of a new feature under pressure [28]: a low $T$ positive contribution with a maximum clearly resolved for $P=67$ and $80 \mathrm{kbar}$. The negative peak of $Q$ centered at $T^{Q_{\min }}$ was not observable at $P=0$ for $H=0$ since $T^{Q_{\min }}<T_{c}$. It emerges rapidly above $T_{c}$ under $P$. Resistivity measurements performed simultaneously show that the pressure dependence of the temperature $T^{\rho_{\max }}$ of the maxima of the resistivity has the same $P$ variation that $T^{Q_{\min }}$. Thus this correlation asserts the same physical origin of $T^{\rho_{\max }}$ and $T^{Q_{\min }}:$ the crossover between high and low temperature paramagnetic regimes. The appearance of a positive peak below $T^{Q_{\min }}$ suggests the occurrence of an antiferromagnetic order since large positive peaks of $Q$ have been observed for well known antiferromagnets like $\mathrm{UCu}_{5}$ [29], TmSe or TmS [30]. In these cases, the change of sign of $Q$ corresponds to the ordering temperature $T_{\mathrm{N}}$. The high magnetic field dependence of the positive contribution of $Q$ [28] is an other evidence of its magnetic origin. As $P$ increases, larger fields are needed to destroy this positive contribution.

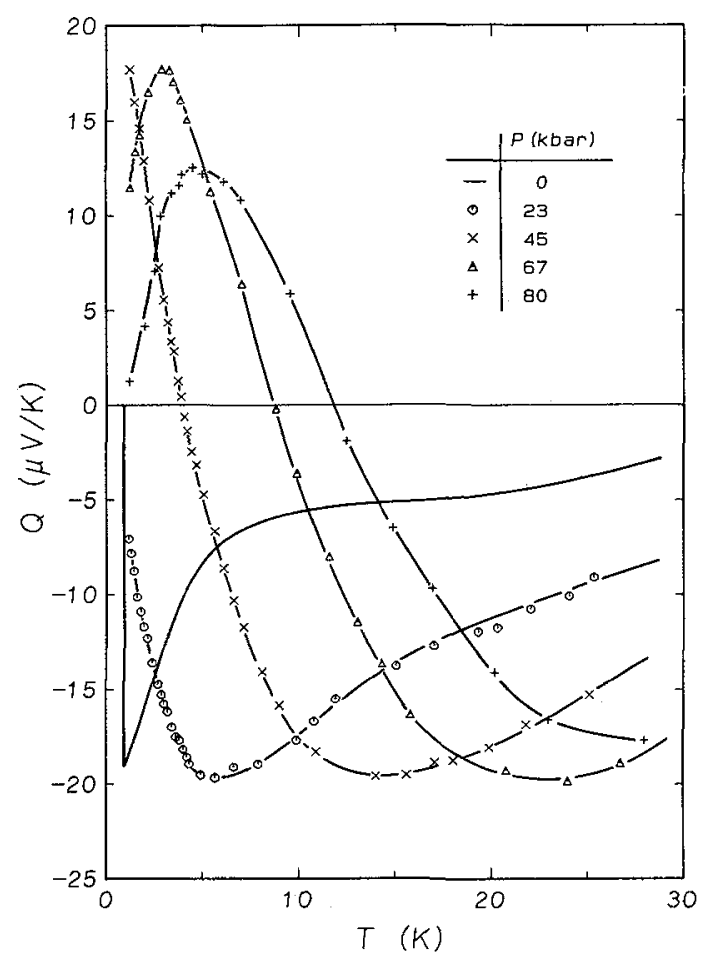

Fig. 3. - Absolute thermoelectric power of $\mathrm{UBe}_{13}$ different pressures. 
From our previous study at $P=0$, a positive peak of $Q$ can be inferred below $150 \mathrm{mK}$. And new specific heat experiments $[1,10]$ under magnetic field lead to the idea that the normal phase (observed through the contribution of the vortices) could be magnetically ordered below $100 \mathrm{mK}$.

The occurrence of magnetic ordering is often detected by a change of regime in the magnetoresistivity at $T_{\mathrm{N}}$, for example, in the cubic magnetic Kondo lattices $\mathrm{CeAl}_{2}\left(T_{\mathrm{N}}=3.8 \mathrm{~K}\right)$ or $\mathrm{CePb}_{3}\left(T_{\mathrm{N}} \sim 1.2 \mathrm{~K}\right)[31$, 32]. Below $T_{N}$, positive contributions to the magnetoresistivity are found while above $T_{N}$, the magnetoresistivity is negative. In $\mathrm{UBe}_{13}$ at $P=0$, the magnetoresistivity has always been found negative (above $\left.T_{\mathrm{c}}(H) !\right)$, down to $35 \mathrm{mK}$ and for $H<21 \mathrm{~T}$ [10]. So $\mathrm{UBe}_{13}$ is paramagnetic in zero pressure for $H>H_{\mathrm{c} 2}$. At $P=45 \mathrm{kbar}$, measurements were performed down to $1.2 \mathrm{~K}$ and only negative magnetoresistivity was observed [28]: $T_{\mathrm{N}}$ should be lower than $1.2 \mathrm{~K}$ for $P \leq 45$ kbar.

But at $\mathrm{P}=67 \mathrm{kbar}$ a positive magnetoresistivity appears at low temperature $(T<4 \mathrm{~K}): T_{\mathrm{N}}$ should then be comparable or higher than $4 \mathrm{~K}$. At this pressure, experiments performed down to $50 \mathrm{mK}$ and up to $H=10 \mathrm{~T}$, show no trace of superconductivity and a large positive residual magnetoresistivity: $\Delta \rho / \rho=32 \%$ at $H=10 \mathrm{~T}$. The $T^{2}$ dependence of $\rho$ was observed below $1 \mathrm{~K}$, with a coefficient $A(67 \mathrm{kbar}) \sim 2 \mu \Omega \mathrm{cmK}^{-2}$ at $H=0$.

Up to now, microscopic experiments $[3,4]$ give no sign of antiferromagnetic order for the superconducting phase of $\mathrm{UBe}_{13}$. It may appear that here superconductivity and antiferromagnetism cannot coexist in contrast to the situation observed where uranium atoms are substituted by thorium atoms $\left(\mathrm{U}_{1-x} \mathrm{Th}_{x} \mathrm{Be}_{13}\right)$ with $x>0.015$ [33]. The $P$ disappearance of superconductivity $\left(T_{\mathrm{c}}(67 \mathrm{kbar})<50 \mathrm{mK}\right)$, may be due to the $P$ increase of the ordering temperature $T_{N}$ of the normal phase. Pressure studies are actually performed to clarify the situation.

At $P=0$, extensive magnetoresistivity experiments were realized down to $35 \mathrm{mK}$ up to $20 \mathrm{~T}$. Below $T=900 \mathrm{mK} \sim T_{\mathrm{c}}(H=0)$, and above $H=4 \mathrm{~T}$, the resistivity can be decomposed in $\rho_{0}(H)$ plus $A(H) T^{2}$ terms [34]. Figure 4 shows the large simultaneous decrease of $A(H)$ and $\rho_{0}(H)$ with $H$; the dashed line shows extrapolated values at low field obtained in order to respect the initial $H^{2}$ dependence of the magnetoresistivity. The interesting phenomena is that the usual scaling (see [1]) between $A(H)$, the differential susceptibility $\chi(H)$ and the linear $T$ term $\gamma(H)$ of the specific heat $(\sqrt{A(H)} \sim \chi(H) \sim \gamma(H))$ breaks down: $\gamma(H)$ and $\chi(H)$ are field independent almost up to $8 \mathrm{~T}[35,10]$ and to $20 \mathrm{~T}[34]$ respectively. This suggests that the field dependence of $A(H)$ is entirely

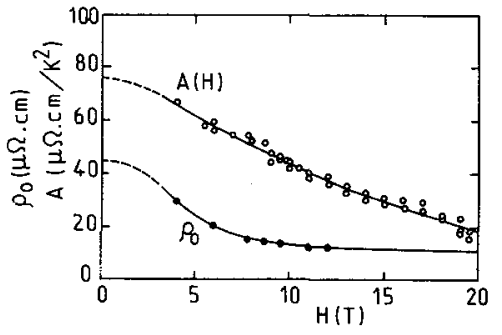

Fig. 4. - Field dependence of (0) $\rho_{0}(H)$ and (0) $A(H)$ of $\mathrm{UBe}_{13}$ at $P=0$. At $P=67 \mathrm{kbar}$ and $H=0, \rho_{0}(0)=$ $32 \mu \Omega \mathrm{cm}$ and $A(0) \sim 2 \mu \Omega \mathrm{cmK}^{-2}$.

due to that of the interactions between quasiparticules, not to the field dependence of "band properties". As a funny check of this hypothesis, one can relate $A(H)$ with superconductivity in analogy with pure aluminium. For $\mathrm{Al}$, the $A$ coefficient is strongly enhanced due to the virtual phonon mediated interaction which is also governing the superconductivity. So in $\mathrm{UBe}_{13}$ the hypothesis made for the origin of the $H$ variation of the $T^{2}$ term of $\rho$ would imply a (known!) field dependence of the attractive potential and thus a field dependent superconducting condensation energy [10].

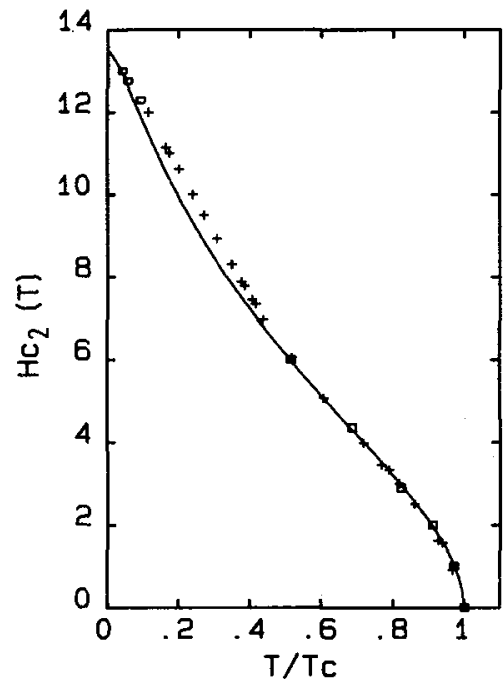

Fig. 5. - For $\mathrm{UBe}_{13}$, comparison of the experimental determination of the upper critical field $H_{\mathrm{c}_{2}}(T)$ with an estimation of $H_{\mathrm{c} 2}(T)$ (full line) assuming an orbital limitation and a BCS condensation energy depending on the magnetic field as described in the text.

Figure 5 shows how the measured unusual shape of the upper critical field $H_{\mathrm{c} 2}(T)$ can be reproduced assuming an orbital limitation and a BCS field depen- 
dent interaction $V(H) \sim \sqrt{A(H)}$ taken from figure 4 [10]. Such an agreement is another strong support to a non phonon mechanism for the superconductivity of $\mathrm{UBe}_{13}$ ! [10]. The fit yields an initial slope of $H_{\mathrm{c} 2}$ at $T_{c}$ of about $180 \mathrm{~T} / \mathrm{K}$, consistent with the experimental estimate of [36] and providing strong support on the possibility of significative critical fluctuation effects near $T_{\mathrm{c}}$ in $\mathrm{UBe}_{13}[10]$.

\section{Conclusion}

Transport experiments are unique by the facility to operate under extreme conditions: high magnetic field, high pressure and small samples. They could be the appropriate measurement to determine the dependence of ground states (notably magnetism) on lattice's imperfections.

At $P=0, \mathrm{CeAl}_{3}$ appears as an antiferromagnet with a metamagnetic transition. Above $P \sim 2 \mathrm{kbar}$, it is a Pauli paramagnet, and no superconducting phase has been observed down to $40 \mathrm{mK}$ and $P<8 \mathrm{kbar}$. At high pressure, $P>23 \mathrm{kbar}, \mathrm{UBe}_{13}$ may be in a normal antiferromagnetic ground state. The low pressure superconducting ground state seems incompatible with antiferromagnetism however the magnetic field may restore this long range order in the vortices.

It seems now admitted (1) that the driving mechanism of heavy fermion superconductors is given by antiferromagnetic couplings. However, the existence of only few cases of superconductors may indicate that, over and above these couplings, specific pairing conditions have to be fulfilled (for example on the wavevector and amplitude of antiferromangetic correlations).

More systematic measurements must be performed to know the importance of disorder on lattice properties: influence on the nature of the ground state, on the magnitude of the effects (size of the ordered moment), on the low temperature excitations... Therefore, special effort must be made to improve the samples quality.

[1] Proceedings of 6th International Conference on crystal field effects and heavy fermion physics, Eds. P. Fulde, B. Lüthi and F. Steglich, J. Magn. Magn. Mater., to be published.

[2] Asayama, K. and Kitaoka, K. this conference and in [1].

[3] Aeppli, G., Bucher, E., Broholm, C., Kjems, K., Goldman, A. and Shirane, G., in [1].

[4] Barth, S., Ott, H. R., Gygax, F. N., Hitti, B., Lippelt, E. and Schenck, A., in [1].

[5] Regnault, L. P., Jacoud, J. L., Rossat Mignod, J., Vettier, C., Lejay, P., Flouquet, J., Walker, E., Jaccard, D. and Amato, A., in [1].
[6] Phillips, N. E., Fisher, R. A., Lacy, S. E., Marcenat, C., Olsen, J. A., Flouquet, J., Amato, A., Jaccard, D., Fisk, Z., Giorgi, A. L., Smith, J. L. and Stewart, G. R., Theoretical and experimental aspects of valence fluctuations and heavy fermions, Eds. L. C. Gupta and S. K. Malik (Plenum Publishing Corporation) 1987, pp. 141150.

[7] Jaccard, D., Cibin, R., Jorda, J. L. and Flouquet, J., Jpn J. Appl. Phys. Suppl. 26 (1987) 517.

[8] Jaccard, D., Cibin, R. and Sierro, J., Helv. Phys. Acta 61 (1988) 530.

[9] Jaccard, D., Cibin, R., Bezinge, A., Sierro, J., Matho, K. and Flouquet, J., in [1].

[10] Brison, J. P., Thesis, Université de Grenoble (1988).

[11] Barth, S., Ott, H. T., Gygax, F. N., Hitti, B. Hitti, Lippelt, E., Schenck, A. and Baines, C., Jpn J. Appl. Phys. 26 (1987) 519.

[12] Sparn, G., Lieke, W., Gottwick, U., Steglich, F. and Grewe, N., J. Magn. Magn. Mater. 47-48 (1985) 521.

[13] See Jaccard, D. and Flouquet, J., Helv. Phys. Acta 60 (1987) 108.

[14] Haen, P., Flouquet, J., Lapierre, F., Lejay, P. and Remenyi, G., J. Low Temp. Phys. 67 (1987) 391.

[15] Andres, K., Graebner, J. E. and Ott, H. R., Phys. Rev. Lett. 35 (1975) 1979.

[16] Usami, K., J. Phys. Soc. Jpn 45 (1977) 466.

[17] Nakamura, H., Kitaoka, Y., Asayama, K. and Flouquet, J., J. Phys. Soc. Jpn, to be published and in [1].

[18] Fierz, C., Thèse Université de Genève (1988); Fierz, C., Jaccard, D., Sierro, J. and Flouquet, J., J. Appl. Phys. 63 (1988) 3899.

[19] Pethick, C. J. and Pines, D., Phys. Rev. Lett. 57 (1986) 118.

[20] Hirshfeld, P., Vollhardt, D. and Wölfe, P., Solid State Commun. 59 (1986) 111.

[21] Schmitt-Rink, S., Miyake, K. and Varma, C. M., Phys. Rev. Lett. 57 (1986) 2575.

[22] Fetisov, E. P. and Khomskii, D. I., JETP Lett. (43) (1986) 432; Sov. Phys. JETP 65 (1987) 59.

[23] Okkawa, F. J., J. Phys. Soc. Jpn 55 (1986) 2527.

[24] Ott, H. R., Rudigier, H., Fisk, Z. and Smith, J. L., Phys. Rev. Lett. 50 (1983) 1595.

[25] Jaccard, D., Flouquet, J., Fisk, Z., Smith, J. L. and Ott, H. R., J. Phys. Lett. France 46 (1985) L811.

[26] Brison, J. P., Ravex, A., Lasjaunias, J. C., Flouquet, J., Jaccard, D., Fisk, Z. and Smith, Proceedings Interlaken Conference, Physica $C$ 153155 (1988) 437. 
[27] Ott, H. R., Rudigier, H., Rice, T. M., Ueda, K., Fisk, Z. and Smith, J. L., Phys. Rev. Lett. 52 (1984) 1915.

[28] Mao, S. Y., Diplôme Université de Genève (1988) Mao, S. Y., Jaccard, D., Sierro, J., Fisk, Z. and Smith, J. L., in [1].

[28] Van Daal, H. J., Buschow, K. H. J., van Aken, P. P. and van Maaren, M. H., Phys. Rev. Lett. 34 (1975) 1457.

[30] Jaccard, D. and Sierro, J., Solid State Commun. 31 (1979) 713.

[31] Lapierre, F., Haen, P., briggs, A. and Sera, M., $J$. Magn. Magn. Mater. 63-64 (1987) 76.
[32] Welp, U., Haen, P., Bruls, G., Remenyi, G., Flouquet, J., Morin, A., Briggs, Cors, G. and Karkut, M., J. Magn. Magn. Mater. 63-64 (1987) 28.

[33] See Fisk, Z., Ott, H. R. and Aeppli, G., Jpn J. Appl. Phys. 26 (1987) 1882.

[34] Remenyi, G., Jaccard, D., Flouquet, J., Briggs, A., Fisk; Z., Smith, J. L. and Ott, H. R., J. Phys. 47 (1986) 3467.

[35] Mayer, H. M., Rauchschwalbe, U., Bredl, C. D., Steglich, F., Rietschel, H., Schmidt, H., Wühl, H. and Beuers, J., Phys. Rev. B 33 (1986) 3168.

[36] Rauchschwalbe, U., Ahlheim, U., Steglich, F., Rainer, D. and Franse, J. J. M., Z. Phys. B 60 (1985) 379. 